\title{
Poor Outcomes in Elderly Kidney Transplant Recipients Receiving Alemtuzumab Induction
}

\author{
Frank P. Hurst ${ }^{a} \quad$ Maria Altieri $^{b}$ Robert Nee ${ }^{a} \quad$ Lawrence Y. Agodoa $^{c}$ \\ Kevin C. Abbott ${ }^{\mathrm{a}}$ Rahul M. Jindald, e \\ ${ }^{\text {a } D e p a r t m e n t ~ o f ~ N e p h r o l o g y, ~ W a l t e r ~ R e e d ~ A r m y ~ M e d i c a l ~ C e n t e r, ~ W a s h i n g t o n, ~ D . C ., ~}{ }^{\text {b }}$ Department of Surgery, \\ Stony Brook University Hospital, Long Island, N.Y., 'NIDDK, National Institutes of Health, Bethesda, Md., and \\ Departments of ${ }^{\mathrm{d}}$ Organ Transplant and ${ }^{\mathrm{e}}$ Surgery, Walter Reed Army Medical Center and \\ George Washington University, Washington, D.C., USA
}

\section{Key Words}

Alemtuzumab · Kidney transplantation • Elderly recipients • Induction agents, complications · Kidney transplants, outcomes

\begin{abstract}
Introduction: Alemtuzumab and rabbit antithymocyte globulin (rATG) are being used with increasing frequency as induction agents in kidney transplantation. Using the US Renal Data Base System, we analyzed the safety profile of these agents in the elderly. Methods: In a cohort of patients transplanted from January 2000 to July 2009 and followed through 2009, we assessed the effect of induction on allograft loss and death among elderly recipients. Recipients were censored at dates of allograft loss, death or the end of study. Independent associations between induction agents and allograft loss or death were examined using multivariate analysis with forward stepwise Cox regression. Results: Among 130,402 patients with first transplants, 14,907 were age 65 years or older. $4,466(30 \%), 3,049(20.5 \%), 1,501(10.1 \%)$, and 999 (6.7\%) were induced with thymoglobulin, basiliximab, daclizumab, and alemtuzumab, respectively. After ad-
\end{abstract}

\section{KARGER}

() 2011 S. Karger AG, Basel

Fax +4161306 1234 E-Mail karger@karger.ch www.karger.com www.karger.com/ajn justing for baseline differences, induction with alemtuzumab was associated with an increased risk of graft loss and death, with an adjusted hazard ratio (AHR) of $1.26(95 \% \mathrm{Cl}$ 1.08-1.48). Risk was also present at other age cutoffs [age $>60$ (AHR 1.16; 95\% Cl 1.03-1.31; $p=0.014$ ), age > 70 (AHR 1.43; $95 \% \mathrm{Cl} 1.13-1.81 ; \mathrm{p}=0.003)$ and age $>75(\mathrm{AHR} 1.68 ; 95 \% \mathrm{Cl}$ 1.07-2.63; $p=0.024)$ ]. Conclusions: In the elderly, alemtuzumab is associated with an escalating risk of death and graft loss in recipients of kidney transplantations.

Copyright $\odot 2011$ S. Karger AG, Basel

\section{Introduction}

The introduction of several potent immunosuppressive agents has improved the outcomes of solid organ transplant; however, success has been tempered by as-

Views expressed in this paper are those of the authors and do not reflect the official policy of the National Institutes of Health, the Department of Army, the Department of Defense, or the United States government. 
sociated complications including potentially life-threatening infections and malignancy [1]. Induction immunosuppression involves intense prophylactic therapy administered at the time of transplantation with the goal of preventing acute rejection and ultimately facilitating a tolerogenic state [2]. The requirement for induction immunosuppression is a consequence of the increased immunogenicity of the allograft in the immediate posttransplant period. Specifically, the cumulative effects of a high frequency of donor-specific T-cell precursors present in most recipients, coupled with innate immune system activation during organ transplantation, account for this vulnerable period for the allograft.

The majority of transplant programs in the US use induction therapy as many of these preparations have been shown to be superior to bolus methylprednisolone when combined with standard maintenance regimens [3]. Most trials use the surrogate endpoint of acute rejection and under these circumstances induction immunosuppression has exhibited a reduction in the incidence of acute rejection when compared to historical standards; however, patient or graft survival have not been clearly shown to improve outcomes [4-6].

The increased use of induction agents in kidney transplantation and given the significant risk of adverse events, in particular with alemtuzumab and rabbit antithymocyte globulin (rATG), we analyzed the US Renal Data Base System (USRDS) with particular emphasis on the cause of death and induction agents. Our analysis is relevant for elderly patients who have decreased immunogenicity and may perhaps not require heavy induction.

\section{Methods}

This study used the USRDS which incorporates extensive baseline and follow-up demographic and clinical data on all patients accessing the Medicare ESRD program in the United States [7]. The variables included in the USRDS standard analysis files (SAFs), as well as methods and validation studies, are published and listed at the USRDS website, under 'Researcher's Guide to the USRDS Database', Section E, 'Contents of all the SAFs'. The demographics of the renal transplant population have been previously described (2011 USRDS report). The files SAF.TXUNOS were used as the primary data set. We used an inception cohort (based on date of transplant) with patients over the age 18 years between January 1, 2000, and July 1, 2009, and followed through to September 30, 2009. Induction and maintenance immunosuppressive medications were determined using data from the TXIRUNOS file. Treated rejection was determined using data from the TXIFUNOS file. Recipients were censored at dates of allograft loss (return to dialysis), time of death, or the end of the study period (September 30, 2009).

Alemtuzumab in Kidney in Elderly

Transplant Recipients
All analyses were performed using SPSS $12.0^{\mathrm{TM}}$ (SPSS, Inc., Chicago, Ill., USA). Files were merged and converted to SPSS files using DBMS/Copy (Conceptual Software, Houston, Tex., USA). Bivariate analysis of factors associated with induction agents was performed with $\chi^{2}$ testing for categorical variables and one-way ANOVA for continuous variables. Statistical significance for bivariate comparisons was defined as $p<0.05$. The independent associations between induction agents and allograft loss or death were examined using multivariate analysis with forward stepwise Cox regression. Variables with $p<0.10$ tested in bivariate analysis for a relationship were entered into multivariate analysis as covariates because of the possibility of negative confounding. Variables thought to have a known clinical association with outcomes were also introduced into multivariate models even if bivariate $p$ values were $>0.10$, in accordance with established principles of model development. Interaction terms were evaluated by adding pairs of significant covariates to the model. Proportionality of hazards was assessed graphically using log minus log plots.

\section{Results}

Among 130,402 patients with first transplants, 14,907 (11.4\%) were age 65 years or older. Excluding any overlap in medications and patients with missing or no induction, 4,466 (30\%), 3,049 (20.5\%), 1,501 (10.1\%), and 999 (6.7\%) were induced with thymoglobulin, basiliximab, daclizumab, and alemtuzumab, respectively. Table 1 demonstrates the differences among older patients with the different induction regimens. Notable differences include more patients of black race, with deceased donors, and delayed graft function (DGF) in the thymoglobulin group, and more expanded criteria donors and higher panel reactive antibody (PRA) in the thymoglobulin and alemtuzumab groups. Maintenance immunosuppression differed in that patients induced with thymoglobulin or alemtuzumab were less likely to be discharged on cyclosporine and steroids.

After adjusting for these differences, induction with alemtuzumab in this population was associated with an increased risk of allograft loss (including death) with an adjusted hazard ratio of 1.26 (95\% CI 1.08-1.48; table 2). This association was not observed with the other induction agents, which can be seen graphically in figure 1 . Other age cutoffs were also assessed and the risk appeared to be present after age $>60$ [adjusted hazard ratio (AHR) 1.16; 95\% CI 1.03-1.31; $\mathrm{p}=0.014]$, with an increasing risk noted for age $\geq 65$ (table 2), age $\geq 70$ (AHR 1.43; 95\% CI 1.13-1.81; $\mathrm{p}=0.003$ ) and age $\geq 75$ (AHR 1.68; 95\% CI $1.07-2.63 ; \mathrm{p}=0.024)$.

After deconstructing the composite outcome of allograft loss, which includes death with functioning al- 
Table 1. Characteristics of older patients (aged $>65$ years) by induction agent

\begin{tabular}{|c|c|c|c|c|c|}
\hline & $\begin{array}{l}\text { Basiliximab } \\
(n=3,049)\end{array}$ & $\begin{array}{l}\text { Daclizumab } \\
(\mathrm{n}=1,501)\end{array}$ & $\begin{array}{l}\text { Thymoglobulin } \\
(\mathrm{n}=4,466)\end{array}$ & $\begin{array}{l}\text { Alemtuzumab } \\
(\mathrm{n}=999)\end{array}$ & $\mathrm{p}$ \\
\hline \multicolumn{6}{|l|}{ Transplant recipient factors } \\
\hline Recipient age, years & $70.0 \pm 3.5$ & $69.9 \pm 3.5$ & $69.8 \pm 3.4$ & $69.9 \pm 3.6$ & 0.024 \\
\hline Male gender vs. female & $2,004(65.7)$ & $988(65.8)$ & $2,731(61.2)$ & $636(63.7)$ & $<0.001$ \\
\hline Black race vs. all others & $432(14.2)$ & $255(17.0)$ & $940(21.0)$ & $146(14.6)$ & $<0.001$ \\
\hline Peak or recent PRA $>20 \%$ & $288(9.9)$ & $188(13.2)$ & $748(17.7)$ & $161(16.8)$ & $<0.001$ \\
\hline Delayed graft function & $515(16.9)$ & $244(16.3)$ & $944(21.1)$ & $140(14.0)$ & $<0.001$ \\
\hline Diabetes & $917(30.1)$ & $476(31.7)$ & $1,429(32.0)$ & $340(34.0)$ & 0.096 \\
\hline Deceased donor & $2,120(69.5)$ & $1,031(68.7)$ & $3,358(75.2)$ & $696(69.7)$ & $<0.001$ \\
\hline Expanded criteria donor & $752(24.7)$ & $363(24.2)$ & $1,300(29.1)$ & $295(29.5)$ & $<0.001$ \\
\hline \multicolumn{6}{|l|}{ Discharge immunosuppression } \\
\hline Tacrolimus & $1,852(60.7)$ & $950(63.3)$ & $3,637(81.4)$ & $840(84.1)$ & $<0.001$ \\
\hline Cyclosporine (Neoral) & $729(23.9)$ & $373(24.9)$ & $400(9.0)$ & $51(5.1)$ & $<0.001$ \\
\hline Mycophenolate mofetil & $2,324(76.2)$ & $1,367(91.1)$ & $3,506(78.5)$ & $692(69.3)$ & $<0.001$ \\
\hline Mycophenolate sodium & 409 (13.4) & $42(2.8)$ & $516(11.6)$ & $84(8.4)$ & $<0.001$ \\
\hline Sirolimus & $283(9.3)$ & $101(6.7)$ & $386(8.6)$ & $15(1.5)$ & $<0.001$ \\
\hline Corticosteroids & $2,594(85.1)$ & $1,327(88.4)$ & $2,849(63.8)$ & $280(28.0)$ & $<0.001$ \\
\hline
\end{tabular}

Data presented as mean \pm standard deviation for continuous variables and $\mathrm{n}(\%)$ for categorical variables.

Table 2. Cox regression analysis of factors associated with allograft loss after renal transplantation among patients aged $>65$ years

\begin{tabular}{lllr}
\hline Variable & AHR & $95 \%$ CI & \multicolumn{1}{c}{$\mathrm{p}$} \\
\hline Induction - basiliximab (ref.) & 1 & & 0.019 \\
Induction daclizumab & 0.961 & $0.859-1.076$ & 0.494 \\
Induction thymoglobulin & 1.031 & $0.940-1.131$ & 0.520 \\
Induction campath & 1.263 & $1.076-1.482$ & 0.004 \\
Recipient age, per year & 1.033 & $1.022-1.045$ & $<0.001$ \\
PRA >20\% & 1.143 & $1.019-1.283$ & 0.023 \\
Year of transplant & 0.948 & $0.929-0.968$ & $<0.001$ \\
Tacrolimus at discharge & 0.720 & $0.645-0.803$ & $<0.001$ \\
Cyclosporine at discharge & 0.880 & $0.778-0.996$ & 0.043 \\
Diabetes mellitus & 1.361 & $1.256-1.474$ & $<0.001$ \\
Deceased donor & 1.367 & $1.237-1.512$ & $<0.001$ \\
Delayed graft function & 1.870 & $1.712-2.041$ & $<0.001$ \\
Male gender & 1.220 & $1.122-1.327$ & $<0.001$ \\
Expanded criteria donor & 1.472 & $1.354-1.600$ & $<0.001$ \\
\hline
\end{tabular}

$\mathrm{AHR}=$ Adjusted hazard ratio; $\mathrm{CI}=$ confidence interval. Included in model but not significant: black race, mycophenolate mofetil, sirolimus, and steroids. lograft, we found that induction with alemtuzumab was associated with an increased risk of death (AHR 1.31; 95\% CI 1.10-1.56; $\mathrm{p}=0.002$ ) in this population (fig. 2). There was no significant association with death-censored allograft loss, which represented the minority of event outcomes ( $\mathrm{n}=321,3.2 \%$ of total). For the patients who had a categorized cause of death available, there were no significant differences noted among cardiac, infectious, or malignant causes of death (table 3).

Higher rejection rates at 1 year were noted among patients induced with basiliximab and alemtuzumab (table 3). As a sensitivity analysis, treated acute rejection in the first year was added to the primary allograft loss model (only including those patients whose allografts survived at least 12 months). Induction with alemtuzumab continued to be associated with increased risk of allograft loss (AHR 1.39; 95\% CI 1.12-1.72; $\mathrm{p}=0.003$ ). In a sensitivity analysis including a subset of elderly patients of lower immunologic risk (low PRA and non-black), similar findings were noted (AHR 1.30; 95\% CI 1.08-1.57; $\mathrm{p}=0.006$ ).

A significant interaction was noted in the model with alemtuzumab and DGF. However, when limiting analysis to only patients with or without DGF, alemtuzumab induction remained significantly associated with al- 


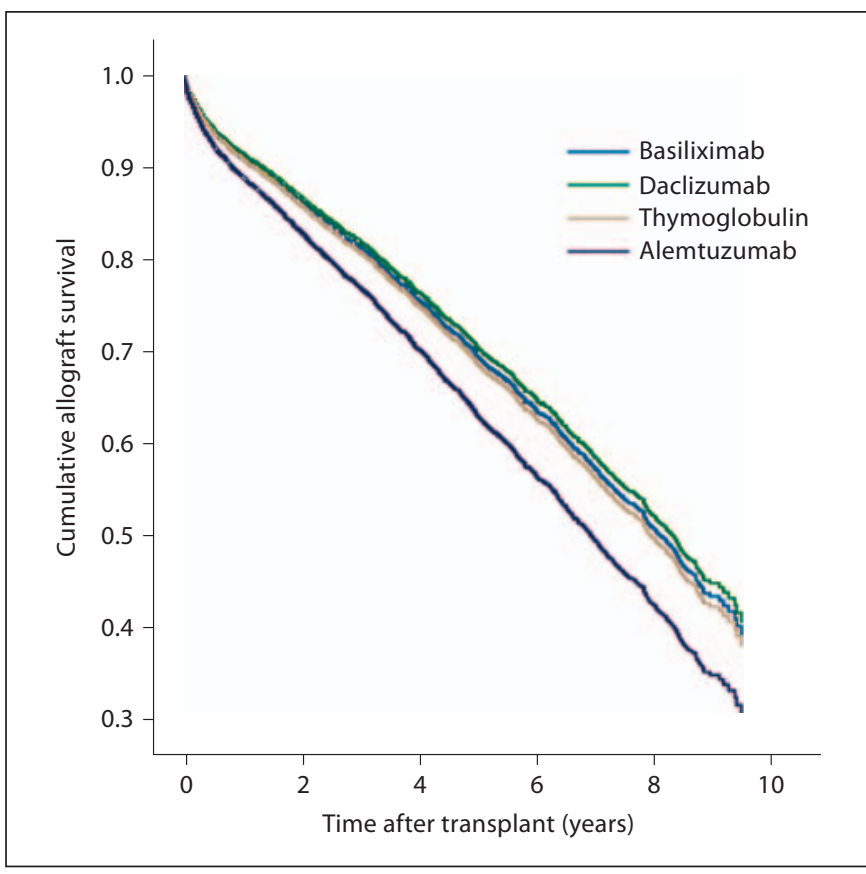

Fig. 1. Time to allograft loss among patients aged $>65$ years with regard to the 4 induction agents.

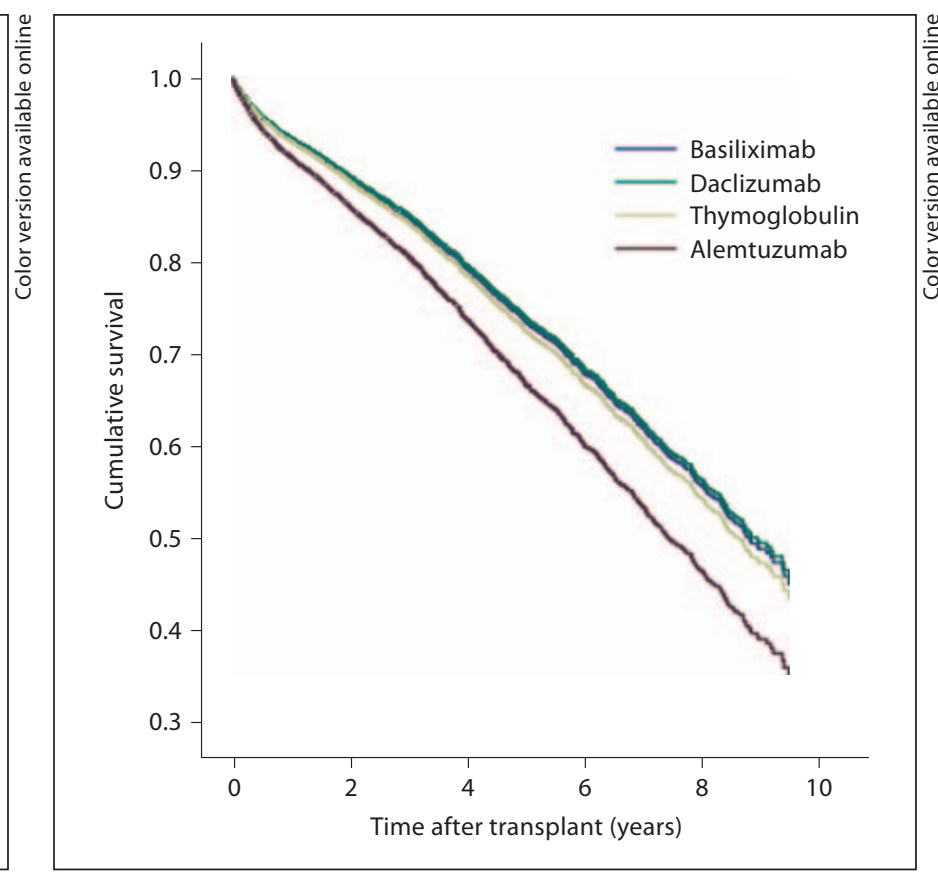

Fig. 2. Time to death among patients aged $>65$ years with regard to the 4 induction agents.

Table 3. Outcomes associated with the 4 induction agents

\begin{tabular}{|c|c|c|c|c|c|}
\hline & $\begin{array}{l}\text { Basiliximab } \\
(\mathrm{n}=3,049)\end{array}$ & $\begin{array}{l}\text { Daclizumab } \\
(\mathrm{n}=1,501)\end{array}$ & $\begin{array}{l}\text { Thymoglobulin } \\
(\mathrm{n}=4,466)\end{array}$ & $\begin{array}{l}\text { Alemtuzumab } \\
(\mathrm{n}=999)\end{array}$ & $\mathrm{p}$ \\
\hline Rejection within 12 months ${ }^{1}$ & $168(6.8)$ & $62(5.1)$ & $170(5.0)$ & $50(6.8)$ & 0.013 \\
\hline Died & 634 & 324 & 679 & 152 & \\
\hline Cause of death missing ( $27.2 \%$ overall $)$ & $252(28.4)$ & $98(23.2)$ & $271(28.5)$ & $46(23.2)$ & 0.090 \\
\hline Cardiac death & $215(33.9)$ & $100(30.9)$ & $199(29.3)$ & $55(36.2)$ & 0.193 \\
\hline Infectious death & $119(18.8)$ & $58(17.9)$ & $150(22.1)$ & $32(21.1)$ & 0.329 \\
\hline Malignancy death & $70(11.0)$ & $43(13.3)$ & $93(13.7)$ & $16(10.5)$ & 0.416 \\
\hline
\end{tabular}

Data presented as n (\%) for categorical variables.

${ }^{1}$ Includes any use of medications to treat rejection within the first year after transplant in those patients whose allograft survived at least 12 months.

lograft loss. When limiting the analysis to patients who were or were not discharged on steroids, there was no significant association between alemtuzumab and the investigated outcomes. However, in a sensitivity analysis which included a subset of elderly patients on both tacrolimus and mycophenolate mofetil, the increased risk of allograft loss (AHR 1.56; 95\% CI 1.23-1.98; p < 0.001) with the use of alemtuzumab in this population remained.

Alemtuzumab in Kidney in Elderly Transplant Recipients

\section{Discussion}

The elderly group ( $>60$ years old) comprises the fasting growing segment of transplant recipients. As of 2008, induction agents were administered in $82 \%$ of kidney recipients, as three types of agents currently account for more than $97 \%$ of induction therapies: rATG, monoclonal antibodies, daclizumab and basiliximab, and alemtuzumab [8]. The recently released data from the OPTN/

Am J Nephrol 2011;34:534-541 
SRTR annual Report (2009) showed that the use of alemtuzumab has steadily increased over the years to $10.7 \%$ of all kidneys transplanted in 2008, while the use of rATG has remained relatively steady at approximately $40 \%$. The use of basiliximab has been steady at approximately $17 \%$ and that of daclizumab has reduced from a peak of $14.4 \%$ in 2001 to $10.9 \%$ in 2008 [9]. Few studies have studied the effect on immunosuppressive agents in the elderly population [10-13] including the increasing use of alemtuzumab $[11,14,15]$.

The present analysis is an extension of the study by Gill et al. [14] which also used the UNOS-based database. Gill et al. [14] showed that alemtuzumab use was associated with a higher risk of acute rejection, death, and allcause graft loss in the elderly population ( $>60$ years old) and that rATG may be preferable in high-risk recipients with high-risk donors and possibly low-risk recipients with high-risk donors. These findings are consistent with the results of our study. In particular, alemtuzumab was associated with an increased risk of graft loss (AHR 1.26) and death (AHR 1.31) among first transplant recipients above 65 years of age. The worse outcomes associated with alemtuzumab versus rATG occurred despite lower proportions of high-risk recipient factors in the alemtuzumab cohort, such as black race, PRA $>20 \%$ and DGF. This association can be potentially explained by the lower rate of steroid use in the alemtuzumab versus rATG group (28 vs. $63.8 \%$ ). However, the higher rate of graft loss in the alemtuzumab group remained significant after adjusting for steroid use.

We also utilized escalating age cutoffs in assessing the risk of graft loss which increased consistently with increasing age in the recipients who received alemtuzumab as an induction agent. Furthermore, this heightened risk of graft loss included death in the analysis, while there was no significant association with death-censored graft loss, suggesting the important contribution of death with functioning graft as an etiology of graft loss. We did not detect any significant differences in the causes of death (cardiac, infections, malignancy) when comparing recipients receiving alemtuzumab with other induction agents, but the cause of death variable was missing in $27 \%$ of cases, so it is difficult to draw any conclusions regarding the etiology of death in this cohort. Given that thymoglobulin is considered to be of similar potency, we were intrigued that this therapy was not also associated with an increased risk of death when compared with the antiIL-2 induction agents. It is possible that if thymoglobulin was used in the elderly population, it was given in lower doses as has been previously reported [11, 15]. Both of these groups concluded that the use of full-dose antithymocyte therapy $(7-10 \mathrm{mg} / \mathrm{kg})$ was associated with increased morbidity in the elderly and low-dose rATG $(<6 \mathrm{mg} / \mathrm{kg})$ is safe and effective to use in patients older that 65 and leads to equivalent graft survival and function without incurring excess morbidity.

We found higher rejection rates at 12 months after transplant among patients induced with basiliximab and alemtuzumab versus rATG (6.8 vs. $5.0 \%$ ). This finding is consistent with outcomes reported by Gill et al. [14] that elderly recipients who received $\mathrm{rATG}$ had the lowest rate of acute rejection within the first year after transplant (7.3\%) compared with the alemtuzumab group (11.4\%). In contrast, Hanaway et al. [16] found a significantly lower rate of biopsy-confirmed acute rejection among patients receiving alemtuzumab than among those receiving conventional induction (basiliximab or rATG) at both 6 ( 3 vs. $15 \%$ ) and 12 months (5 vs. 17\%). However, rates of late biopsy-confirmed acute rejection (occurring between 12 and 36 months after transplant in patients who did not have rejection within the first 12 months) were higher in the alemtuzumab versus conventional groups (8 vs. $3 \%$; $\mathrm{p}=0.03)$. Antibody-mediated rejection was not an end point in the study and donor-specific antibody formation was not evaluated and therefore, humoral rejection cannot be excluded.

In our study, we did not detect any significant interactions between high-risk recipient factors (i.e. black race and PRA $>20 \%$ ) and alemtuzumab for the outcome of death. Therefore, pooled, rather than stratified analyses were performed to assess mortality rate in the study. Failure to detect significant differences in stratified analyses in other studies may represent insufficient sample sizes.

Alemtuzumab [17] was approved by the US Food and Drug Administration in 1999 as a treatment for lymphoid malignancy (Campath-1H; Genzyme, Cambridge, Mass., USA). Alemtuzumab is an anti-CD52 humanized, complement-fixing, IgG1 monoclonal antibody that targets primarily T cells, but also B cells, macrophages, NK cells, and some granulocytes and leads to antibody-dependent lysis. The Cambridge group performed the first clinical trial of alemtuzumab induction with cyclosporine monotherapy in kidney transplant recipients, and reported outcomes comparable to historical controls [18]. Kirk et al. [19] investigated the use of alemtuzumab in a pilot tolerance trial designed to test the requirement for maintenance immunosuppression. Although there was significant depletion of peripheral lymphocytes and monocytes, all patients developed rejection episodes 
and required commencement of maintenance immunosuppression. In the first randomized, prospective trial evaluating alemtuzumab, efficacy appeared similar to induction with either thymoglobulin or daclizumab; of interest, most of the patients in the alemtuzumab arm remained steroid-free [20]. Ciancio et al. [21] carried out a parallel, open-label, randomized controlled trial comparing the following three regimens: alemtuzumab, rATG, and daclizumab induction in patients who received deceased donor renal grafts. After a 15-month follow-up, there were similar rates of acute rejection and other adverse events in the 3 groups, and $80 \%$ of the alemtuzumab patients remained steroid-free. After 36 months, once more there were similar rates of acute rejection and other adverse events [21].

In a recent publication, Hanaway et al. [16] compared induction therapy with alemtuzumab to basiliximab or rAGT in a patient population at either high or low immunologic risk over a 3 -year period. Patients were stratified according to acute rejection risk (repeat transplant, a peak or current value of panel-reactive antibodies of $>20 \%$ or black race). The group concluded that biopsyconfirmed acute rejection at 3 years in the low-risk group was lower with alemtuzumab than with basiliximab ( 10 vs. $22 \%, p=0.003$ ), but in the high-risk group, there was no difference when comparing alemtuzumab with rATG (18 vs. $15 \%, \mathrm{p}=0.63$ ). Adverse-event rates were similar among all 4 treatment groups; however, the incidence of serious adverse events related to cancer was higher in the alemtuzumab group $(\mathrm{p}=0.03)$. Amongst the low-risk patients, the rate of serious infectious adverse events was lower with basiliximub versus alemtuzumab ( 22 vs. $35 \%, p=0.02)$. Among high-risk patients, the rate of all infectious adverse events was slightly higher with rATG versus alemtuzumab (81 vs. $60 \%$, p = 0.009) [16].

In another study comparing infection rates, Kaufman et al. [22] showed that alemtuzumab $(\mathrm{n}=123)$ and basiliximab $(n=155)$ induction in patients undergoing early glucocorticoid withdrawal showed no significant differences. They found that 1-year actual patient and death-censored kidney transplant survival rates were 96.8 versus $99.4 \%$, and 99.2 versus $99.4 \%$, respectively ( $\mathrm{p}=$ n.s.). The rate of acute rejection for both groups was equivalent (alemtuzumab 14.9\%; basiliximab 13.5\%), and there was no difference in renal function at 1 year. Also, long-term patient and graft survival did not differ between the 2 groups, but alemtuzumab had a lower rate of early rejection, defined as $<3$ months [22]. Farney et al. [23] compared alemtuzumab and rATG induction in adult kidney and pancreas transplantation using similar maintenance immunosuppression. Of the 98 patients, 77 (79\%) underwent kidney alone (KA) transplant, 17 (17\%) pancreas-kidney transplant, and 4 (4\%) pancreas after kidney transplant. Of the $77 \mathrm{KA}$ transplants, 66 (86\%) were from deceased donors and 31 (40\%) from expanded criteria donors. Patient and kidney survival were 100 and 96\%, respectively. It was concluded that survival and overall acute rejection rates were similar between alemtuzumab and rATG groups; however, acute rejection occurred in $9(20 \%)$ rATG patients compared with $0(0 \%)$ alemtuzumab patients who received KA transplants $(p=0.007)$ [23]. In a later publication, the authors concluded that both therapies were equally safe, but alemtuzumab was associated with less biopsy-proven acute rejection [13].

Several studies have compared the incidence rates of rejection and patient and graft-survival rates $[12,13,16$, 18, 22-25] and use in African American and Hispanic populations [26], but thus far few studies have looked into the efficacy and safety in the patient population over 65 years $[11,14,15]$. This is important, as the use of alemtuzumab has increased over the past decade in the United States and the fact that alemtuzumab is such an efficacious agent and its mechanism of depletion is so profound that it may take up to a year after administration for the immune system to regain full function. Significant adverse-effect profile includes neutropenia, thrombocytopenia, anemia, nausea, vomiting, diarrhea, headache, among others, which may require the administration of corticosteroids, acetaminophen, and antihistamine agents in order to decrease the severity of the adverse effects $[24,27]$.

Currently, the literature shows that rATG appears to be a superior agent in the prevention of biopsy-proven acute rejections, though the graft and patient survivals are not significant. There were are no differences in overall survival and biopsy-proven acute rejection rates between alemtuzumab and rATG groups; however, acute rejection occurred in $9(20 \%)$ rATG patients compared with 0 alemtuzumab patients who received KA transplants $(p=0.007)$ [23]. In a multicenter study, rATG was compared to daclizumab. The study selected highrisk HLA-sensitized patients and randomly assigned them to either rATG $(n=227)$ or daclizumab group $(n=227)$. The study showed that compared to the daclizumab group, patients treated with rATG had a lower incidence of steroid-resistant rejection (2.7 vs. $14.9 \%$; $\mathrm{p}=0.002)$ and biopsy-proven acute rejection (15 vs. $27.2 \% ; \mathrm{p}=0.016)$ at 1 year, and patient survival and graft 
survival were similar [28]. Another multicenter study compared basiliximab and rATG. The study followed 135 recipients who were in either the basiliximab group $(n=70)$ versus rATG $(n=65)$. Basiliximab had low acute rejection rates similar to those achieved by rATG; however, it had a better safety profile, with no increase in malignancies, infections, or deaths [29]. Another study showed that the 6-year patient and graft survival in the rATG group was not significantly higher than that compared to basiliximab ( 91.7 vs. $85 \%$ and 89.7 vs. $83.6 \%$, respectively); however, cytomegalovirus infections were more prevalent in the rATG group ( 22 vs. $5 \%, \mathrm{p}=0.05)$ and so was the incidence of hematological complications [30].

The Cochrane review looked into the comparison of IL2Ra treatment to rATG in 18 studies (1,844 participants) and found that there were similar results in graft loss or acute rejection, but there was a benefit of rATG therapy over IL2Ra for biopsy-proven acute rejection at 1 year (9 studies; RR 1.30; 95\% CI 1.01-1.67), but the side effects of rATG were much higher compared to the IL2Ra induction agents, as a 75\% increase in malignancy (7 studies; RR 0.25 ; 95\% CI 0.07-0.87) and a 32\% increase in cytomegalovirus infections (4 studies; MD-11.20 $\mu \mathrm{mol} / \mathrm{l}$; $95 \%$ CI -19.94 to -2.09 ). In addition, the review concluded that there was a higher incidence of fever, cytokine release and leucopenia [7].

The limitations of the USRDS are well described in the literature [24]. Pertinent limitations were the inability to determine the precise cause of increased graft loss or patient death using alemtuzumab. Another limitation is the lack of dosing of medications in the USRDS.

We have shown that induction with alemtuzumab in elderly patients was associated with an increased risk of graft loss and death. It may be speculated that transplant programs recognizing the profound depleting effects of alemtuzumab may empirically be reducing the dosage of maintenance immunosuppression. Historically, alemtuzumab has allowed a reduction or total avoidance of steroids in recipients of kidney transplantation. Ongoing clinical trials will clarify this; until then, we urge caution in using alemtuzumab in elderly patients.

\section{References}

$\checkmark 1$ Fishman JA: Infection in solid-organ transplant recipients. N Engl J Med 2007;357: 2601-2614.

-2 Hawksworth JS, Leeser D, Jindal RM, Falta E, Tadaki D, Elster EA: New directions for induction immunosuppression strategy in solid organ transplantation. Am J Surg 2009; 197:515-524.

-3 Shapiro R, Young JB, Milford EL, Trotter JF, Bustami RT, Leichtman AB: Immunosuppression: evolution in practice and trends, 1993-2003. Am J Transplant 2005;5:874886.

-4 Meier-Kriesche HU, Schold JD, Kaplan B: Long-term renal allograft survival: have we made significant progress or is it time to rethink our analytic and therapeutic strategies? Am J Transplant 2004;4:1289-1295.

5 Kirk AD: Induction immunosuppression. Transplantation 2006;82:593-602.

6 Szczech LA, Berlin JA, Feldman HI: The effect of antilymphocyte induction therapy on renal allograft survival. A meta-analysis of individual patient-level data. Anti-Lymphocyte Antibody Induction Therapy Study Group. Ann Intern Med 1998;128:817-826.

7 Webster AC, Ruster LP, McGee R, et al: Interleukin 2 receptor antagonists for kidney transplant recipients. Cochrane Database Syst Rev 2010;1:CD003897.

-8 Markmann JF, Fishman JA: Alemtuzumab in kidney-transplant recipients. N Engl J Med 2011;364:1968-1969.
9 Annual Report of the US Organ Procurement and Transplantation Network and the Scientific Registry of Transplant Recipients: Transplant Data 1999-2008. Rockville, US Department of Health and Human Services, Health Resources and Services Administration, Healthcare Systems Bureau, Division of Transplantation, 2009.

10 Meier-Kriesche HU, Ojo AO, Hanson JA, Kaplan B: Exponentially increased risk of infectious death in older renal transplant recipients. Kidney Int 2001;59:1539-1543.

11 Laftavi MR, Patel S, Soliman MR, et al: Lowdose thymoglobulin use in elderly renal transplant recipients is safe and effective induction therapy. Transplant Proc 2011;43: 466-468.

12 Huang E, Cho YW, Hayashi R, Bunnapradist $S$ : Alemtuzumab induction in deceased donor kidney transplantation. Transplantation 2007;84:821-828.

13 Farney AC, Doares W, Rogers J, et al: A randomized trial of alemtuzumab versus antithymocyte globulin induction in renal and pancreas transplantation. Transplantation 2009;88:810-819.

14 Gill J, Sampaio M, Gill JS, et al: Induction immunosuppressive therapy in the elderly kidney transplant recipient in the United States. Clin J Am Soc Nephrol 2011;6:1168-1178.
15 Patel SJ, Knight RJ, Suki WN, et al: Rabbit antithymocyte induction and dosing in deceased donor renal transplant recipients over 60 yr of age. Clin Transplant 2011;25:E250E256.

16 Hanaway MJ, Woodle ES, Mulgaonkar S, et al: Alemtuzumab induction in renal transplantation. N Engl J Med 2011;364:1909-1919.

17 Jacobs SA, Foon KA: Monoclonal antibody therapy of leukaemias and lymphomas. Expert Opin Biol Ther 2005;5:1225-1243.

18 Calne R, Moffatt SD, Friend PJ, et al: Campath IH allows low-dose cyclosporine monotherapy in 31 cadaveric renal allograft recipients. Transplantation 1999;68:1613-1616.

19 Kirk AD, Hale DA, Mannon RB, et al: Results from a human renal allograft tolerance trial evaluating the humanized CD52-specific monoclonal antibody alemtuzumab (CAMPATH-1H). Transplantation 2003;76: 120-129.

20 Ciancio G, Burke GW, Gaynor JJ, et al: A randomized trial of three renal transplant induction antibodies: early comparison of tacrolimus, mycophenolate mofetil, and steroid dosing, and newer immune-monitoring. Transplantation 2005;80:457-465.

21 Ciancio G, Gaynor JJ, Roth D, et al: Randomized trial of thymoglobulin versus alemtuzumab (with lower dose maintenance immunosuppression) versus daclizumab in living donor renal transplantation. Transplant Proc 2010;42:3503-3506. 
-22 Kaufman DB, Leventhal JR, Axelrod D, Gallon LG, Parker MA, Stuart FP: Alemtuzumab induction and prednisone-free maintenance immunotherapy in kidney transplantation: comparison with basiliximab induction - long-term results. Am J Transplant 2005;5:2539-2548.

-23 Farney A, Sundberg A, Moore P, et al: A randomized trial of alemtuzumab vs. anti-thymocyte globulin induction in renal and pancreas transplantation. Clin Transplant 2008; 22:41-49.

-24 Ciancio G, Burke GW 3rd: Alemtuzumab (Campath-1H) in kidney transplantation. Am J Transplant 2008;8:15-20.
25 Ortiz J, Palma-Vargas J, Wright F, et al: Campath induction for kidney transplantation: report of 297 cases. Transplantation 2008;85: 1550-1556.

26 Ciancio G, Burke GW, Gaynor JJ, et al: Campath-1H induction therapy in African American and Hispanic first renal transplant recipients: 3-year actuarial follow-up. Transplantation 2008;85:507-516.

27 Morris PJ, Russell NK: Alemtuzumab (Campath-1H): a systematic review in organ transplantation. Transplantation 2006;81: 1361-1367.
28 Noel C, Abramowicz D, Durand D, et al: Daclizumab versus antithymocyte globulin in high-immunological-risk renal transplant recipients. J Am Soc Nephrol 2009;20:13851392.

29 Sollinger H, Kaplan B, Pescovitz MD, et al: Basiliximab versus antithymocyte globulin for prevention of acute renal allograft rejection. Transplantation 2001;72:19159191.

30 Ulrich F, Niedzwiecki S, Pascher A, et al: Long-term outcome of ATG vs. Basiliximab induction. Eur J Clin Invest 2011;41:971978. 